\title{
Recurrent Chylothorax Caused by a Retained Guidewire in a Central Vein: A Case Report
}

\author{
Young-Do Jeon ${ }^{1,2}$, Eun Kyoung Kim², Ji-Won Hwang ${ }^{1}$, Il Park ${ }^{1}$, Nam-Joon Kim ${ }^{1}$ \\ ${ }^{1}$ Division of Cardiology, Department of Medicine, Samsung Medical Center, Sungkyunkwan University School of Medicine, Seoul, \\ ${ }^{2}$ Division of Cardiology, Department of Medicine, Iksan Hospital, Iksan, Korea
}

Corresponding Author:

Young-Do Jeon, MD

Division of Cardiology, Department of Medicine, Iksan Hospital, 969 Muwang-ro, Iksan 54543, Korea

Tel: $+82-63-840-9143$

Fax: $+82-63-840-9458$

E-mail: jyd82@naver.com

Received: July 20, 2017

Revised: September 15, 2017

Accepted: September 21, 2017
The loss of a guidewire occurs very rarely during central venous catheterization (CVC). Guidewire loss can lead to serious mechanical, thrombotic, and infectious complications. A 74-year-old woman was admitted to our institution with dyspnea. Chest computed tomography revealed a guidewire that had remained in a central vein for 3 years, and the patient had extensive central venous thrombosis and a chylothorax. The guidewire and venous thrombus could not be removed because of high perioperative risk due to adhesions and chronic atrophic changes of the intravascular layer. The chylothorax was resistant to conservative treatment. Therefore, the patient was subjected to thoracic duct ligation and embolization. Subsequently, the chylothorax disappeared and the patient was discharged. Physicians should be especially aware of the adverse effects caused by a remnant guidewire and the need for simple chest radiography after CVC.

Key Words: Chylothorax, Central venous catheterization, Venous thrombosis

\section{INTRODUCTION}

A chylothorax results from leakage of lymphatic fluid, and is caused by obstruction or injury of the thoracic duct". Nontraumatic chylothorax results from increased superior vena cava pressure due to malignancy, lymphangioleiomyomatosis, sarcoidosis, cirrhosis, tuberculosis, or thrombosis causing venous obstruction ${ }^{2,3}$. As the correction of underlying causes is the first and best treatment option for nontraumatic chylothorax, the causative factors should be evaluated.

The loss of a guidewire occurs very rarely during central venous catheterization $(\mathrm{CVC})^{4-6)}$. The guidewire may remain unnoticed in the vessel for a long time and may not induce specific symptoms ${ }^{6}$. However, it may cause vascular injury, cardiac injury, arrhythmia, or chronic venous thrombosis? The development of a chylothorax following venous thrombosis caused by a retained guidewire after catheterization has not been reported. We report a case of recurrent chylothorax caused by chronic total central venous obstruction due to a retained guidewire after CVC.

\section{CASE REPORT}

A 74-year-old woman was admitted because of a femoral fracture. A large left pleural effusion was incidentally detected on preoperative chest radiography (Fig. 1). We performed diagnostic thoracentesis and drained milky-chylous fluid. Pleural fluid analysis revealed a white blood cell count of $>1,000 / \mathrm{LL}$ (87\% lymphocyte), glucose $124 \mathrm{mg} / \mathrm{dL}$, protein $3,522 \mathrm{mg} / \mathrm{dL}$, amylase $32 \mathrm{U} / \mathrm{L}$, adenosine deaminase $11.5 \mathrm{IU} / \mathrm{L}$, and triglyceride $295 \mathrm{mg} / \mathrm{dL}$. The pleural fluid culture showed no growth. The pleural effusion was diagnosed as a chylothorax, although there was no history of chest trauma.

Chest computed tomography revealed the presence of a wire and chronic thrombus from the right internal jugular

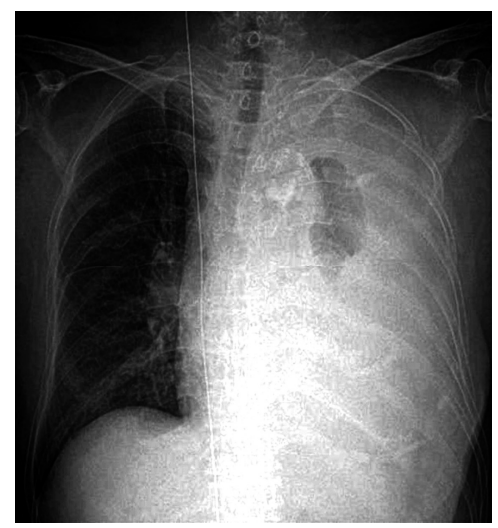

Fig. 1. Initial chest radiograph. Left pleural effusion and linear structure across the right lung were observed. 

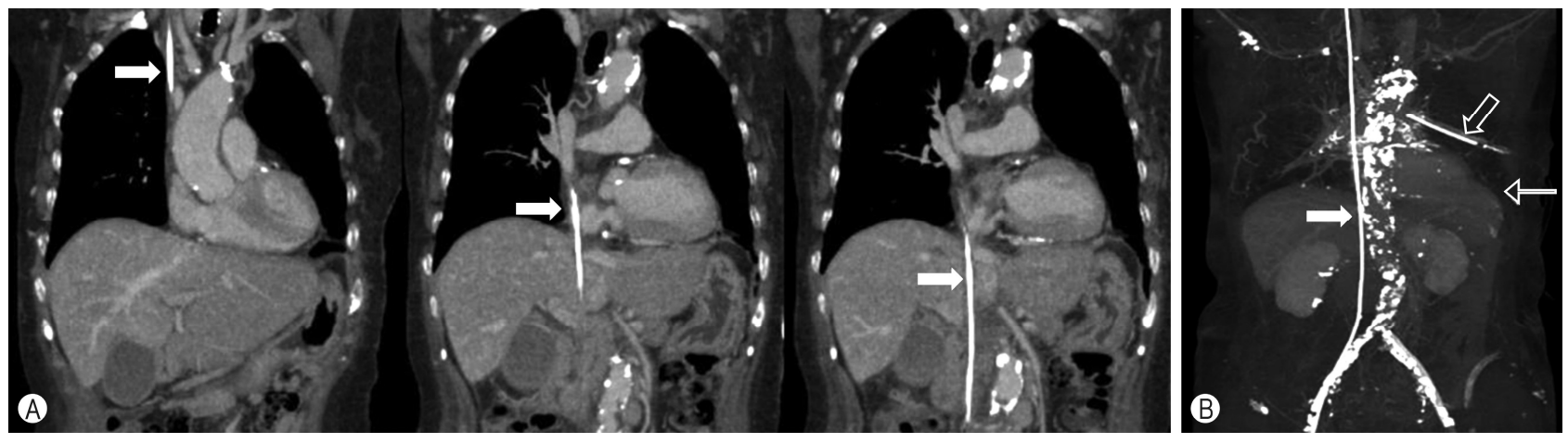

Fig. 2. Chest computed tomographic angiography. The retained wire and diffusely collapsed central vein with thrombus were observed. (A) The white arrows indicate the guidewire along the central vein. (B) Maximal intensity projection view: The black arrow indicates the chest tube. The white arrow indicates the guidewire and the black arrow indicates the chest tube.

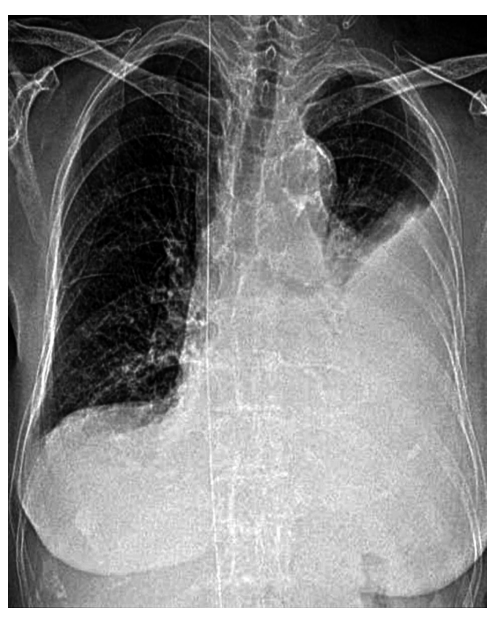

Fig. 3. Chest radiograph 1 year after the first visit. The recurrent left pleural effusion was observed.

vein to a lower extremity vein (Fig. 2A, B). Review of the initial chest radiographic image also confirmed the wire in the central vein. Two years prior, she was hospitalized in the intensive care unit of another hospital with ischemic colitis. At that time, CVC was performed via the subclavian vein, but the guidewire was not removed after the procedure. The wire remained in the vessel for 2 years between the right internal jugular and femoral vein. We determined that the chylothorax was caused by obstruction of a central vein by chronic thrombus. However, removal of the retained wire was dangerous because the thrombus chronically and totally obstructed a long segment of the central venous structure and was adherent to the vessel wall. Therefore, after conservative treatment for chylothorax, the patient was placed on anticoagulation therapy and discharged.

One year later, she revisited the Emergency Department with new-onset dyspnea. Physical examination was unremarkable, except for decreased breath sounds in the bilateral lower lobes. Chest radiography showed recurrent pleural effusion (Fig. 3). Examination of the pleural fluid also revealed a chylothorax (total white blood cell count $>1,000 / \mu \mathrm{L}(78 \%$
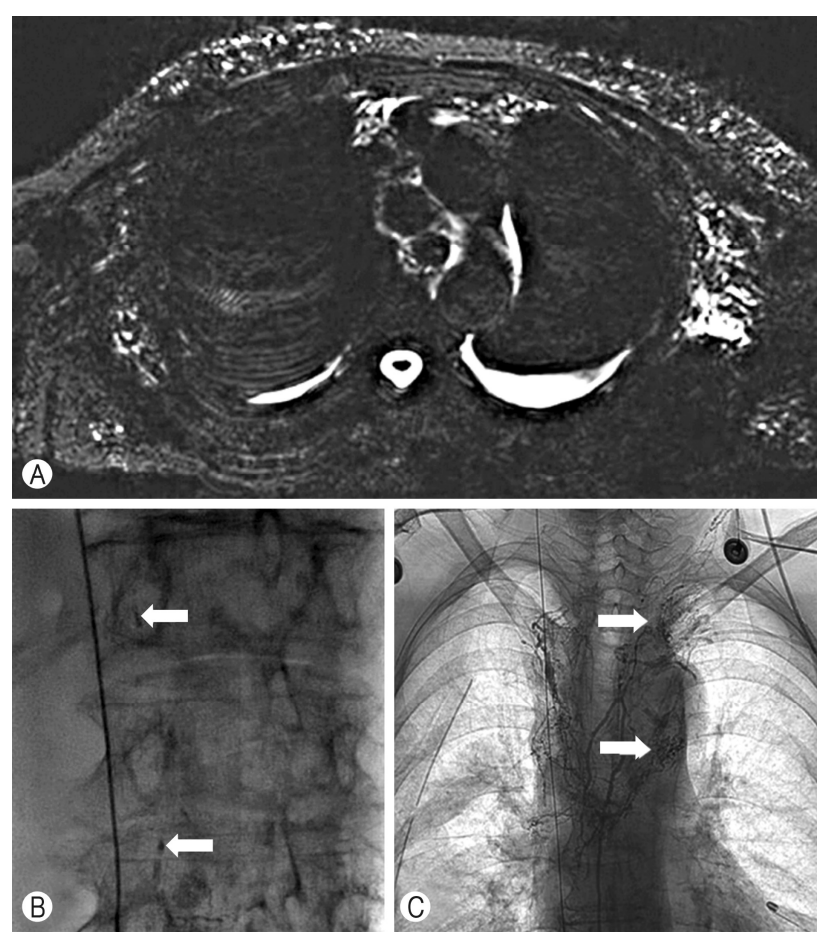

Fig. 4. Magnetic resonance lymphangiography of the thorax. (A) Heavily T2-weighted image: The leakage at the left side of the aortic arch and chylous fluid collection in the bilateral pleural space were observed. (B) The distal thoracic duct was obstructed. The white arrows indicate obstructed thoracic duct. (C) The white arrows indicates abnormal collateral circulation of lymphatic vessels.

lymphocytes), glucose $123 \mathrm{mg} / \mathrm{dL}$, protein 3,518 mg/dL, amylase $36 \mathrm{U} / \mathrm{L}$, cholesterol $59 \mathrm{mg} / \mathrm{dL}$, and triglyceride $635 \mathrm{mg} / \mathrm{dL}$ ). Octreotide was administered to reduce lymph flow, and total parenteral nutrition and water restriction were started. Percutaneous catheter drainage was continued for 2 weeks, but the amount of chylothorax did not decrease. Magnetic resona- 
nce lymphangiography revealed collapse of the thoracic duct and central lymphatic system. Leakage of lymphatic fluid was also suspected (Fig. 4A). In addition, imaging showed obstruction of the distal thoracic duct with abnormal collateral channels in both the hilum and apex of the lung (Fig. 4B, C). She underwent thoracoscopic-thoracic duct ligation and embolization of the thoracic duct and collateral channels. The guidewire and venous thrombus were not removed because of high perioperative risk from adhesions and chronic atrophic intravascular changes. The chylothorax was not observed at the 6-month follow-up after discharge, and the patient is followed as an outpatient in good general condition.

\section{DISCUSSION}

Chylothorax is a life-threatening complication of chronic venous obstruction. When chylothorax is diagnosed and is resistant to conservative treatment, the possibility of extensive venous occlusion should be suspected, and surgical treatment should be considered. Here, we present a case of recurrent chylothorax caused by chronic total obstruction of the long segment of the central vein due to a retained guidewire. Loss of guidewire occurs very rarely during $\mathrm{CVC}^{8-10}$. However, it can cause severe complications similar to those reported in our case, unless the wire is removed immediately. Remnant wire not only occupies the vessel space but also obstructs venous circulation, leading to complications including arrhythmia, cardiac or vascular perforation, looping of wire, and thrombus ${ }^{4,11)}$. If thrombus in the central vein is left untreated, unexpected adverse events may occur, including chylothorax ${ }^{8)}$. Early detection of a retained wire following thrombus formation is key to complete resolution after wire removal and anticoagulation.

The clinical success of chylothorax treatment depends on the underlying cause. Tamai et al. ${ }^{9)}$ reported a case of chylothorax associated with thrombosis in the superior vena cava that was resistant to thoracic duct ligation. A stent was implanted in the superior vena cava, after which the chylothorax disappeared. Manghat et al. ${ }^{10)}$ reported a case of chylothorax secondary to extensive central venous thrombosis. They performed catheter-directed thrombolysis. In these cases, the venous thrombus was discovered in a relatively acute phase compared with that in our case. In our case, conservative medical treatment failed to prevent a chylothorax. Therefore, thoracic duct ligation and embolization were selected as the first treatment option ${ }^{12)}$. However, because the retained guidewire and chronic venous thrombosis from the jugular vein to the femoral vein could not be removed, thoracic duct ligation and embolization were only palliative for prevention of recurrent chylothorax.

By reviewing this case, we hope that physicians will gain sufficient awareness of the long-term adverse events of guidewire placement and the need for early detection of this complication using simple chest radiography after CVC.

Conflicts of Interest Disclosures: The researchers claim no conflicts of interest.

\section{REFERENCES}

1. Schild HH, Strassburg CP, Welz A, Kalff J. Treatment options in patients with chylothorax. Dtsch Arztebl Int 2013;110: 819-26.

2. Huggins JT. Chylothorax and cholesterol pleural effusion. Semin Respir Crit Care Med 2010;31:743-50.

3. Bender B, Murthy V, Chamberlain RS. The changing management of chylothorax in the modern era. Eur J Cardiothorac Surg 2016;49:18-24.

4. Srivastav R, Yadav V, Sharma D, Yadav V. Loss of guide wire: a lesson learnt review of literature. J Surg Tech Case Rep 2013; 5:78-81.

5. Gunduz Y, Vatan MB, Osken A, Cakar MA. A delayed diagnosis of a retained guidewire during central venous catheterisation: a case report and review of the literature. BMJ Case Rep 2012 Nov 19;2012. pii: bcr2012007064. https://doi.org/10.1136/ bcr-2012-007064.

6. Auweiler M, Kampe S, Zähringer M, Buzello S, von Spiegel T, Buzello W, et al. The human error: delayed diagnosis of intravascular loss of guidewires for central venous catheterization. J Clin Anesth 2005; 17:562-4.

7. Khasawneh FA, Smalligan RD. Guidewire-related complications during central venous catheter placement: a case report and review of the literature. Case Rep Crit Care 2011;2011:287261.

8. Hsu HF, Chou YH, Wang CR, Wu SC. Catheter-related superior vena cava syndrome complicated by chylothorax in a premature infant. Chang Gung Med J 2003;26:782-6.

9. Tamai A, Kurishima C, Seki M, Masutani S, Taketazu M, Senzaki $H$. Stent implantation for effective treatment of refractory chylothorax due to superior vena cava obstruction as a complication of congenital cardiac surgery. Clin Med Insights Cardiol 2012;6:97-101.

10. Manghat N, Hancock J, Walsh M, Puckett M, Noble R, Travis S. Thrombolysis for central venous occlusion causing bilateral chylothorax in a patient with down syndrome. J Vasc Interv Radiol 2004;15:511-5.

11. Seo IS, Choi JH, Nam YH, Im JW, Whang HK, Won KH. A case of left brachiocephalic vein total occlusion due to acute thrombosis soon after permanent pacemaker insertion. J Korean Geriatr Soc 2007;11:229-33.

12. Talwar A, Lee HJ. A contemporary review of chylothorax. Indian J Chest Dis Allied Sci 2008;50:343-51. 